\title{
Minimal Total Dominating Functions of Corona Product Graph of a Path with a Star
}

\author{
M.Siva Parvathi \\ Dept. of Mathematics, K.R.K. Govt. Degree \\ College, Addanki-523201, Andhra Pradesh, India
}

\author{
B.Maheswari \\ Dept. of Applied Mathematics, S.P.Women's \\ University, Tirupat-517502, Andhra Pradesh, India
}

\begin{abstract}
Graph theory is one of the most flourishing branches of modern mathematics and computer applications. Domination in graphs has been studied extensively in recent years and it is an important branch of graph theory. An introduction and an extensive overview on domination in graphs and related topics is surveyed and detailed in the two books by Haynes et al. $[6,7]$. Recently dominating functions in domination theory have received much attention. In this paper we present some results on minimal signed dominating functions and minimal total signed dominating functions of corona product graph of a path with a star.
\end{abstract}

\section{Keywords}

Corona Product, signed dominating function, Total signed dominating function

\section{INTRODUCTION}

Domination Theory has a wide range of applications to many fields like Engineering, Communication Networks, Social sciences, linguistics, physical sciences and many others. Allan, R.B. and Laskar, R.[1], Cockayne, E.J. and Hedetniemi, S.T. [2] have studied various domination parameters of graphs.

Recently, dominating functions in domination theory have received much attention. The concepts of total dominating functions and minimal total dominating functions are introduced by Cockayne et al. [3]. Jeelani Begum, S. [8] has studied some total dominating functions of Quadratic Residue Cayley graphs.

Frucht and Harary [5] introduced a new product on two graphs $\mathrm{G} 1$ and $\mathrm{G} 2$, called corona product denoted by $\mathrm{G} 1 \odot \mathrm{G} 2$. The object is to construct a new and simple operation on two graphs G1 and G2 called their corona, with the property that the group of the new graph is in general isomorphic with the wreath product of the groups of G1 and of G2.

The authors have studied some dominating functions of corona product graph of a cycle with a complete graph [9] and published papers on minimal dominating functions, some variations of $\mathrm{Y}$ - dominating functions and $\mathrm{Y}$ - total dominating functions $[10,11,12]$.

In this paper we study the concept of total dominating functions of corona product graph of a path with a star and some results on minimal total dominating functions are obtained.

\section{CORONA PRODUCT OF $P_{n}$ AND $K_{1 m}$}

The corona product of a path $\mathbb{P}_{\mathrm{n}}$ with star $K_{1 \mathrm{~m}}$ is a graph obtained by taking one copy of a $n-$ vertex path $\mathbb{P}_{\mathrm{n}}$ and $n$ copies of $K_{1 \mathrm{~m}}$ and then joining the $i^{\text {th }}$ vertex of $P_{\mathrm{n}}$ to every vertex of $i^{\text {th }}$ copy of $K_{1, n}$ and it is denoted by $P_{82} \odot K_{1} m^{x}$

We require the following theorem whose proof can be found in Siva Parvathi, M. [8].

Theorem 2.1: The degree of a vertex $\mathbb{V}_{\mathfrak{i}}$ in $G=P_{\mathrm{n}} \odot K_{1 \mathrm{~m}}$ is given by

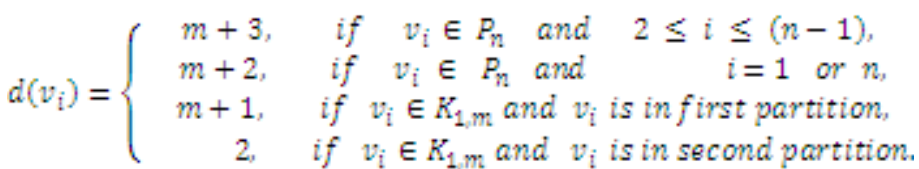

\section{TOTAL DOMINATING SETS AND TOTAL DOMINATING FUNCTIONS}

The concepts of total dominating functions and minimal total dominating functions are introduced by Cockayne et al. [4]. In this section we prove some results related to total dominating functions of the graph $G=P_{\mathrm{r}} \odot K_{1 \mathrm{~m}}$. First let us recall some definitions.

Definition: Let $G\left(V_{a} E\right)$ be a graph without isolated vertices. A subset $T$ of $V$ is called a total dominating set (TDS) if every vertex in $V$ is adjacent to at least one vertex in $T$.

If no proper subset of $T$ is a total dominating set, then $T$ is called a minimal total dominating set (MTDS) of $G$.

Definition: The minimum cardinality of a MTDS of $G$ is called a total domination number of $G$ and is denoted by $v_{L}(G)$.

Theorem 3.1: The total domination number of $G=P_{\mathrm{g}} \odot R_{1, \mathrm{~m}}$ is $n$

Proof: Let $\mathrm{T}$ denote a total dominating set of $\mathrm{G}$. Let $\mathrm{T}$ contain the vertices of the path $P_{\text {rn }}$.

By the definition of the graph $\mathrm{G}$, every vertex in $P_{\mathrm{n}}$ is adjacent to all vertices of associated copy of $K_{1, \mathrm{~m}}$. That is the vertices in $P_{n}$ dominate the vertices in all copies of $K_{1 \mathrm{~m}}$ respectively. Further these vertices being in $\mathbb{P}_{\mathrm{m}}$, they dominate among themselves. Thus T becomes a TDS of G. Obviously this set is minimum. 
Therefore $\eta_{t}(G)=n$

Theorem 3.2: Let $\mathrm{T}$ be a MTDS of $G=P_{\mathrm{n}} \odot K_{1 \mathrm{gx}}$ Then a function $f: V \rightarrow\left[0_{w} 1\right]$ defined by

$f(v)= \begin{cases}1, & \text { if } v \in T, \\ 0, & \text { otherwise. }\end{cases}$

becomes a MTDF of $G=P_{\mathrm{g} 2} \odot R_{1 \mathrm{~m}}$

Proof: Consider the graph $G=P_{\mathrm{g}} \odot K_{1 \mathrm{~m}}$ with vertex set V.

Let $\mathrm{T}$ be a MTDS of $G$ such that it contain all the vertices of $\mathrm{P}_{\mathrm{n}}$.

The summation value taken over $W(\mathbb{D})$ of $\mathbb{D} \in \mathbb{V}$ is as follows:

Case 1: Let $\mathbb{D} \in \mathcal{P}_{\mathrm{g}}$ be such that $d(\mathbb{D})=m+3$ in $G$.

Then $M(\mathbb{v})$ contains $m+1$ vertices of $K_{1} m$ and two vertices of $P_{\mathrm{y}}$ in $G_{x}$

So $\sum_{u \in N(v)} f(u)=1+1+[\underbrace{0+\ldots \ldots+0}_{(m+1) \text {-times }}]=2$.

Case 2: Let $\mathbb{D} \in P_{\mathrm{n}}$ be such that $d(\mathbb{D})=m+2$ in $G_{x}$

Then $N(\mathbb{E})$ contains $m+1$ vertices of $R_{1}$ and one vertex of $P_{\mathrm{n}}$ in $G_{\mathrm{x}}$

So $\sum_{u \in N(v)} f(u)=1+[\underbrace{0+\ldots \ldots+0}_{(m+1) \text {-times }}]=1$.

Case 3: Let $\mathbb{V} \in R_{1 m}$ be such that $d(\mathbb{D})=m+1$ in $G_{x}$

Then $N(\mathbb{D})$ contains $m$ vertices of $K_{1, m}$ and one vertex of $P_{y}$ in $G_{x}$

So $\sum_{u \in N(v)} f(u)=1+[\underbrace{0+\ldots \ldots+0}_{m \text {-times }}]=1$.

Case 4: Let $\mathbb{v} \in K_{1 \mathrm{~m}}$ be such that $d(\mathbb{D})=2$ in $G_{x}$

Then $M(v)$ contains one vertex of $K_{1, n}$ and one vertex of $P_{\text {\% }}$ in $G_{x}$

So $\sum_{u \in N(v)} f(u)=1+0=1$.

Therefore for all possibilities,

we get $\sum_{u \in N(v)} f(u) \geq 1, \quad$ w $v \in \mathbb{V}$.

This implies that $\mathrm{f}$ is a TDF.

Now we check for the minimality of $f$.

Define $g: V \rightarrow[0,1]$ by $g(v)= \begin{cases}\mathrm{r}, & \text { for } \mathrm{v}=\mathrm{v}_{\mathrm{k}} \in T, \\ 1, & \text { for } \mathrm{v} \in \mathrm{T}-\left\{\mathrm{v}_{\mathrm{k}}\right\}, \\ 0, & \text { otherwise. }\end{cases}$

where $0<\mathrm{r}<1$.

Since strict inequality holds at the vertex $v_{k} \in T$, it follows that $\mathrm{g}<\mathrm{f}$.

Case (i): Let $\mathbb{v} \in \mathcal{P}_{\mathrm{n}}$ be such that $d(\mathbb{v})=m+3$ in $\mathrm{G}$.

Sub case 1: Let $v_{k} \in N(v)$.

Then

$$
\sum_{u \in N(v)} g(u)=r+1+[\underbrace{0+\ldots \ldots+0}_{(m+1) \text {-times }}]=r+1>1 \text {. }
$$

Sub case 2: Let $v_{k} \notin N(v)$.

Then $\sum_{u \in N(v)} g(u)=1+1+[\underbrace{0+\ldots \ldots+0}_{(m+1) \text {-times }}]=2$.

Case (ii): Let $\mathbb{v} \in P_{\mathrm{n}}$ be such that $d(\mathbb{D})=m+2$ in $\mathrm{G}$.

Sub case 1: Let $v_{k} \in N(v)$.

Then $\sum_{u \in N(v)} g(u)=r+[\underbrace{0+\ldots \ldots . .0}_{(m+1) \text {-times }}]=r<1$.

Sub case 2: Let $v_{k} \notin N(v)$.

Then $\sum_{u \in N(v)} g(u)=1+[\underbrace{0+\ldots \ldots .+0}_{(m+1) \text {-times }}]=1$.

Case (iii): Let $\mathbb{v} \in K_{1, m}$ be such that $d(\mathbb{v})=m+1$ in $\mathrm{G}$.

Sub case 1: Let $v_{k} \in N(v)$.

Then $\sum_{u \in N(v)} g(u)=r+[\underbrace{0+\ldots \ldots+0}_{m \text {-times }}]=r<1$.

Sub case 2: Let $v_{k} \notin N(v)$.

Then $\sum_{u \in N(v)} g(u)=1+[\underbrace{0+\ldots \ldots+0}_{m \text {-times }}]=1$.

Case (iv): Let $\mathbb{V} \in K_{1 \mathrm{~m}}$ be such that $d(\mathbb{v})=2$ in $\mathrm{G}$.

Sub case 1: Let $v_{k} \in N(v)$.

Then $\sum_{u \in N(v)} g(u)=r+0=r<1$.

Sub case 2: Let $v_{k} \notin N(v)$.

Then $\sum_{u \in N(v)} g(u)=1+0=1$. 
This implies that $\sum_{u \in N(v)} g(u)<1$, for some $\mathbb{E} \in V$.

So $g$ is not a TDF.

Since $g$ is taken arbitrarily, it follows that there exists no $g<f$ such that $g$ is a TDF.

Therefore $f$ is a MTDF.

Theorem 3.3: A function $f_{x} V \rightarrow[0,1]$ defined by $f(v)=\frac{1}{q}, \forall \in V$ is a TDF of $G=P_{x} \odot K_{1, w}$ if $q \leq 2$. It is a MTDF if $q=2$.

Proof: Consider the graph $G=P_{\mathrm{g}} \odot K_{1 \mathrm{~m}}$ with vertex set V.

Let $f$ be a function defined as in the hypothesis.

Case I: Suppose $\mathrm{q}<2$.

Case 1: Let $\mathbb{D} \in \mathcal{P}_{\mathrm{n}}$ be such that $d(\mathbb{v})=m+3$ in $G_{x}$

So $\sum_{u \in N(v)} f(u)=\underbrace{\frac{1}{q}+\frac{1}{q}+\ldots \ldots .+\frac{1}{q}}_{(m+3) \text {-times }}=\frac{m+3}{q}>1$,

since $q<2$ and $m \geq 2$.

Case 2: Let $\mathbb{E} \in P_{n}$ be such that $d(\mathbb{D})=m+2$ in $G_{x}$

So $\sum_{u \in N(v)} f(u)=\underbrace{\frac{1}{q}+\frac{1}{q}+\ldots \ldots .+\frac{1}{q}}_{(m+2) \text {-times }}=\frac{m+2}{q}>1$,

since $\mathrm{q}<2$ and $\mathrm{m} \geq 2$.

Case 3: Let $\mathbb{D} \in K_{1}$ be such that $d(\mathbb{D})=m+1$ in $\mathrm{G}$.

So $\sum_{u \in N(v)} f(u)=\underbrace{\frac{1}{q}+\frac{1}{q}+\ldots \ldots .+\frac{1}{q}}_{(m+1) \text {-times }}=\frac{m+1}{q}>1$,

since $\mathrm{q}<2$ and $\mathrm{m} \geq 2$.

Case 4: Let $\mathbb{D} \in K_{1 \mathrm{~m}}$ be such that $\mathbb{A}(\mathbb{D})=2$ in $G_{x}$

So $\sum_{u \in N(v)} f(u)=\frac{1}{q}+\frac{1}{q}=\frac{2}{q}>1$, since $\mathrm{q}<2$.

Therefore for all possibilities,

we get $\sum_{u \in N(v)} f(u)>1$, $\forall \in W_{a}$

This implies that $f$ is a TDF.

Now we check for the minimality of $f$.

Define $g: V \rightarrow[0,1]$ by

$g(v)= \begin{cases}r, & \text { for } v=v_{k} \in V, \\ \frac{1}{q}, & \text { otherwise. }\end{cases}$ where $0<7<\frac{1}{q}$.

Since strict inequality holds at a vertex $\mathbb{v}_{\mathbb{k}}$ of $\mathrm{V}$, it follows that $g<f$.

Case (i): Let $\mathbb{D} \in P_{n n}$ be such that $d(\mathbb{v})=m+3$ in $\mathrm{G}$.

Sub case 1: Let $v_{k} \in N(v)$.

$$
\text { Then } \begin{aligned}
\sum_{u \in N(v)} g(u) & =r+\underbrace{\frac{1}{q}+\frac{1}{q}+\ldots \ldots+\frac{1}{q}}_{(m+2)-\text { times }} \\
& <\frac{1}{q}+\frac{m+2}{q}=\frac{m+3}{q}>1,
\end{aligned}
$$

since $\mathrm{q}<2$ and $\mathrm{m} \geq 2$.

Sub case 2: Let $v_{k} \notin N(v)$.

Then $\sum_{u \in N(v)} g(u)=\underbrace{\frac{1}{q}+\frac{1}{q}+\ldots \ldots+\frac{1}{q}}_{(m+3) \text {-times }}=\frac{m+3}{q}>1$, since $\mathrm{q}<2$ and $\mathrm{m} \geq 2$.

Case (ii): Let $\mathbb{D} \in \mathcal{P}_{n}$ be such that $d(\mathbb{D})=m+2$ in $\mathrm{G}$.

Sub case 1: Let $v_{k} \in N(v)$.

$$
\text { Then } \begin{aligned}
\sum_{u \in N(v)} g(u) & =r+\underbrace{\frac{1}{q}+\frac{1}{q}+\ldots \ldots+\frac{1}{q}}_{(m+1)-\text { times }} \\
& <\frac{1}{q}+\frac{m+1}{q}=\frac{m+2}{q}>1,
\end{aligned}
$$

since $\mathrm{q}<2$ and $\mathrm{m} \geq 2$.

Sub case 2: Let $v_{k} \notin N(v)$.

Then $\sum_{u \in N(v)} g(u)=\underbrace{\frac{1}{q}+\frac{1}{q}+\ldots \ldots .+\frac{1}{q}}_{(m+2) \text {-times }}=\frac{m+2}{q}>1$, since $\mathrm{q}<2$ and $\mathrm{m} \geq 2$.

Case (iii): Let $\mathbb{D} \in K_{1 \mathrm{~m}}$ be such that $d(\mathbb{D})=m+1$ in $\mathrm{G}$.

Sub case 1: Let $v_{k} \in N(v)$.

$$
\text { Then } \begin{aligned}
\sum_{u \in N(v)} g(u)= & r+\underbrace{\frac{1}{q}+\frac{1}{q}+\ldots \ldots+\frac{1}{q}}_{m-\text { times }} \\
& <\frac{1}{q}+\frac{m}{q}=\frac{m+1}{q}>1,
\end{aligned}
$$

since $\mathrm{q}<2$ and $\mathrm{m} \geq 2$.

Sub case 2: Let $v_{k} \notin N(v)$. 
So $\sum_{u \in N(v)} g(u)=\underbrace{\frac{1}{q}+\frac{1}{q}+\ldots \ldots . .+\frac{1}{q}}_{(m+1) \text {-times }}=\frac{m+1}{q}>1$,

since $\mathrm{q}<2$ and $\mathrm{m} \geq 2$.

Case (iv): Let $\mathbb{D} \in K_{1 \mathrm{~m}}$ be such that $d(\mathbb{D})=2$ in G.

Sub case 1: Let $v_{k} \in N(v)$

Then $\sum_{u \in N(v)} g(u)=r+\frac{1}{q}<\frac{2}{q}$.

Since $\mathrm{q}<2$, it follows that $\frac{2}{q}>1$.

Sub case 2: Let $v_{k} \notin N(v)$.

Then $\sum_{u \in N(v)} g(u)=\frac{1}{q}+\frac{1}{q}=\frac{2}{q}>1$, since $\mathrm{q}<2$.

Hence, it follows that $\sum_{u \in N(v)} g(u)>1, \quad \forall v \in \mathbb{v}$.

Thus $g$ is a TDF.

This implies that $f$ is not a MTDF.

Case II: Suppose $\mathrm{q}=2$.

Case 5: Let $\mathbb{v} \in \mathcal{P}_{\mathrm{n}}$ be such that $d(\mathbb{v})=m+3$ in $G_{x}$

So

$\sum_{u \in N(v)} f(u)=\underbrace{\frac{1}{q}+\frac{1}{q}+\ldots \ldots .+\frac{1}{q}}_{(m+3)-\text { times }}=\frac{m+3}{q}=\frac{m+3}{2}=1+\frac{m+1}{2}>1$.

Case 6: Let $\mathbb{D} \in \mathcal{P}_{\mathrm{n}}$ be such that $\mathrm{d}(\mathbb{v})=m+2$ in $\mathrm{G}$.

So

$\sum_{u \in N(v)} f(u)=\underbrace{\frac{1}{q}+\frac{1}{q}+\ldots \ldots .+\frac{1}{q}}_{(m+2)-\text { times }}=\frac{m+2}{q}=\frac{m+2}{2}=1+\frac{m}{2}>1$.

Case 7: Let $\mathbb{E} \in K_{1 m}$ be such that $d(\mathbb{E})=m+1$ in $\mathrm{G}$.

So

$\sum_{u \in N(v)} f(u)=\underbrace{\frac{1}{q}+\frac{1}{q}+\ldots \ldots+\frac{1}{q}}_{(m+1) \text {-times }}=\frac{m+1}{q}=\frac{m+1}{2}>1$,

since $m \geq 2$.

Case 8: Let $\mathbb{V} \in K_{1 \mathrm{~m}}$ be such that $d(\mathbb{E})=2$ in $\mathrm{G}$.

So $\sum_{u \in N(v)} f(u)=\frac{1}{q}+\frac{1}{q}=\frac{2}{q}=\frac{2}{2}=1$.

Therefore for all possibilities,

we get $\sum_{u \in N(v)} f(u) \geq 1$, ษึ $\mathbb{w} \in \mathbb{W}$.

This implies that $f$ is a TDF.

Now we check for the minimality of $f$.
Define $g: V \rightarrow[0,1]$ by

$g(v)= \begin{cases}r, & \text { for } v=v_{k} \in V, \\ \frac{1}{q}, & \text { otherwise. }\end{cases}$

where $0<8<\frac{1}{q}$.

Since strict inequality holds at a vertex $\mathbb{V}_{\mathrm{k}}$ of $\mathrm{V}$, it follows that $g<f$.

Then as in case (i), for $\mathbb{D} \in P_{\mathrm{n}}$ be such that $d(\mathbb{D})=m+3$, we get

$$
\begin{aligned}
& \sum_{u \in N(v)} g(u)=r+\underbrace{\frac{1}{q}+\frac{1}{q}+\ldots \ldots .+\frac{1}{q}}_{(m+2)-\text { times }}>1, \\
& \text { if } v_{k} \in N[v] .
\end{aligned}
$$

And

$\sum_{u \in N(v)} g(u)=\underbrace{\frac{1}{q}+\frac{1}{q}+\ldots \ldots . .+\frac{1}{q}}_{(m+3) \text {-times }}=\frac{m+3}{q}=\frac{m+3}{2}=1+\frac{m+1}{2}>1$,

if $v_{k} \notin N[v]$.

Again as in case (ii), for $\mathbb{v} \in P_{\mathrm{n}}$ be such that $d(\mathbb{D})=m+2$,

We have $\sum_{u \in N(v)} g(u)=r+\underbrace{\frac{1}{q}+\frac{1}{q}+\ldots \ldots+\frac{1}{q}}_{(m+1)-\text { times }}>1$,

if $v_{k} \in N[v]$.

And

$\sum_{u \in N(v)} g(u)=\underbrace{\frac{1}{q}+\frac{1}{q}+\ldots \ldots . .+\frac{1}{q}}_{(m+2) \text {-times }}=\frac{m+2}{q}=\frac{m+2}{2}=1+\frac{m}{2}>1$,

if $v_{k} \notin N[v]$.

Again we can see as in case (iii) that for $\mathbb{D} \in \mathbb{K}_{1, \mathrm{~m}}$ be such that $d(v)=m+1_{2}$

$$
\sum_{u \in N(v)} g(u)=r+\underbrace{\frac{1}{q}+\frac{1}{q}+\ldots \ldots . .+\frac{1}{q}}_{m \text {-times }}>1,
$$

if $v_{k} \in N[v]$.

And $\sum_{u \in N(v)} g(u)=\underbrace{\frac{1}{q}+\frac{1}{q}+\ldots \ldots .+\frac{1}{q}}_{(m+1) \text {-times }}=\frac{m+1}{q}=\frac{m+1}{2}>1$,

if $v_{k} \notin N[v]$.

Similarly we can show as in case (iv) that $\mathbb{D} \in \mathbb{K}_{1, \mathrm{~m}}$ be such that $d(v)=2$, 
$\sum_{u \in N(v)} g(u)=r+\frac{1}{q}<\frac{2}{q}=\frac{2}{2}=1$, if $v_{k} \in N[v]$,

and $\sum_{u \in N(v)} g(u)=\frac{1}{q}+\frac{1}{q}=\frac{2}{q}=\frac{2}{2}=1$, if $v_{k} \notin N[v]$.

This implies that $\sum_{u \in N(v)} g(u)<1$, for some $\mathbb{v} \in V$.

So $g$ is not a TDF.

Since $g$ is defined arbitrarily, it follows that there exists no $g<f$ such that $g$ is a TDF.

Thus $f$ is a MTDF.

Theorem 3.4: A function $f: V \rightarrow[0,1]$ defined by $f(v)=\frac{p}{q}, \forall w \in V$ where $p=\min (m, n) \quad$ and $q=\max (m, n)$ is a TDF of $G=P_{n} \odot K_{1 n}$ if $\frac{p}{q} \geq \frac{1}{2}$. Otherwise it is not a TDF. Also it becomes a MTDF if $\frac{p}{9}=\frac{1}{2}$.

Proof: Consider the graph $G=P_{\mathrm{g}} \odot K_{1 \mathrm{~m}}$ with vertex set V.

Let $f: V \rightarrow[0,1]$ be defined by $f(\mathbb{D})=\frac{F^{p}}{q} \quad \forall \mathbb{V} \in V$, where $p=\min (m, n)$ and $q=\max (\operatorname{mn} n)$.

Clearly $\frac{p}{q}>0$.

Case 1: Let $\mathbb{D} \in \mathcal{P}_{\mathrm{g} \text { }}$ be such that $d(\mathbb{D})=m+3$ in $G_{x}$

So $\sum_{u \in N(v)} f(u)=\underbrace{\frac{p}{q}+\frac{p}{q}+\ldots \ldots . .+\frac{p}{q}}_{(m+3) \text {-times }}=(m+3) \frac{p}{q}$.

Case 2: Let $\mathbb{D} \in \mathcal{P}_{\mathrm{m}}$ be such that $d(\mathbb{D})=m+2$ in $G_{x}$

So $\sum_{u \in N(v)} f(u)=\underbrace{\frac{p}{q}+\frac{p}{q}+\ldots \ldots .+\frac{p}{q}}_{(m+2) \text {-times }}=(m+2) \frac{p}{q}$.

Case 3: Let $\mathbb{v} \in K_{1, m}$ be such that $d(\mathbb{v})=m+1$ in $G_{a}$

So $\sum_{u \in N(v)} f(u)=\underbrace{\frac{p}{q}+\frac{p}{q}+\ldots \ldots . .+\frac{p}{q}}_{(m+1) \text {-times }}=(m+1) \frac{p}{q}$.

Case 4: Let $\mathbb{D} \in K_{1 \mathrm{~m}}$ be such that $d(\mathbb{D})=2$ in $G_{x}$

So $\sum_{u \in N(v)} f(u)=\frac{p}{q}+\frac{p}{q}=2\left(\frac{p}{q}\right)$.

From the above four cases, we observe that $f$ is a TDF if $\frac{p}{q} \geq \frac{1}{2}$

Otherwise $f$ is not a TDF.

Case 5: Suppose $\frac{p}{q}>\frac{1}{2}$.

Clearly $f$ is a TDF.

Now we check for the minimality of $f$.
Define $g: V \rightarrow[0,1]$ by

$g(v)= \begin{cases}\mathrm{r}, & \text { if } \mathrm{v}=\mathrm{v}_{1} \in V, \\ \frac{\mathrm{p}}{\mathrm{q}}, & \text { otherwise. }\end{cases}$

where $0<\mathrm{g}<\frac{\mathrm{p}}{\mathrm{q}}$.

Since strict inequality holds at a vertex $\mathbb{E}_{\mathrm{R}}$ of $\mathrm{V}$, it follows that $\theta<f$

Case (i): Let $\mathbb{D} \in P_{n}$ be such that $d(\mathbb{D})=m+3$ in $\mathrm{G}$.

Sub case 1: Let $v_{k} \in N(v)$.

$$
\text { Then } \begin{aligned}
\sum_{u \in N(v)} g(u) & =r+\underbrace{\frac{p}{q}+\frac{p}{q}+\ldots \ldots+\frac{p}{q}}_{(m+2) \text {-times }} \\
& <\frac{p}{q}+(m+2) \frac{p}{q}=(m+3) \frac{p}{q}>1,
\end{aligned}
$$

since $\frac{p}{q}>\frac{1}{2}$ and $m \geq 2$.

Sub case 2: Let $v_{k} \notin N(v)$.

Then $\sum_{u \in N(v)} g(u)=\underbrace{\frac{p}{q}+\frac{p}{q}+\ldots \ldots .+\frac{p}{q}}_{(m+3) \text {-times }}=(m+3) \frac{p}{q}>1$,

since $\frac{p}{q}>\frac{1}{2}$ and $m \geq 2$.

Case (ii): Let $\mathbb{v} \in \mathcal{P}_{\mathrm{n}}$ be such that $d(\mathbb{v})=m+2$ in $\mathrm{G}$.

Sub case 1: Let $v_{k} \in N(v)$.

$$
\text { Then } \begin{aligned}
\sum_{u \in N(v)} g(u)= & r+\underbrace{\frac{p}{q}+\frac{p}{q}+\ldots \ldots .+\frac{p}{q}}_{(m+1)-\text { times }} \\
& <\frac{p}{q}+(m+1) \frac{p}{q}=(m+2) \frac{p}{q}>1,
\end{aligned}
$$

since $\frac{p}{q}>\frac{1}{2}$ and $m \geq 2$.

Sub case 2: Let $v_{k} \notin N(v)$.

Then $\sum_{u \in N(v)} g(u)=\underbrace{\frac{p}{q}+\frac{p}{q}+\ldots \ldots .+\frac{p}{q}}_{(m+2)-\text { times }}=(m+2) \frac{p}{q}>1$,

since $\frac{p}{q}>\frac{1}{2}$ and $m \geq 2$.

Case (iii): Let $\mathbb{D} \in K_{1 \mathrm{~m}}$ be such that $d(\mathbb{v})=m+1$ in $\mathrm{G}$.

Sub case 1: Let $v_{k} \in N(v)$.

Then $\sum_{u \in N(v)} g(u)=r+\underbrace{\frac{p}{q}+\frac{p}{q}+\ldots \ldots . .+\frac{p}{q}}_{m \text {-times }}$ 


$$
<\frac{p}{q}+m \frac{p}{q}=(m+1) \frac{p}{q}>1,
$$

since $\frac{p}{q}>\frac{1}{2}$ and $m \geq 2$.

Sub case 2: Let $v_{k} \notin N(v)$.

Then $\sum_{u \in N(v)} g(u)=\underbrace{\frac{p}{q}+\frac{p}{q}+\ldots \ldots .+\frac{p}{q}}_{(m+1)-\text { times }}=(m+1) \frac{p}{q}>1$, since $\frac{p}{q}>\frac{1}{2}$ and $m \geq 2$.

Case (iv): Let $\mathbb{v} \in K_{1, n}$ be such that $\mathbb{L}(\mathbb{v})=2$ in G.

Sub case 1: Let $v_{k} \in N(v)$.

Then $\sum_{u \in N(v)} g(u)=r+\frac{p}{q}<2\left(\frac{p}{q}\right)$.

Since $\frac{p}{q}>\frac{1}{2}$ and $m \geq 2$ it follows that $2\left(\frac{p}{q}\right)>1$.

Sub case 2: Let $v_{k} \notin N(v)$.

Then $\sum_{u \in N(v)} g(u)=\frac{p}{q}+\frac{p}{q}=2\left(\frac{p}{q}\right)>1$, since $\frac{p}{q}>\frac{1}{2}$.

Hence it follows that $\sum_{u \in N(v)} g(u)>1$, $\mathbb{w} \in \mathbb{W}$.

Thus for all possibilities, we get that $g$ is a TDF.

This implies that $f$ is not a MTDF.

Case 6: Suppose $\frac{p}{q}=\frac{1}{2}$.

As in case 1 and 2, we have that

$$
\sum_{u \in N(v)} f(u)=\underbrace{\frac{p}{q}+\frac{p}{q}+\ldots \ldots .+\frac{p}{q}}_{(m+3)-\text { times }}=(m+3)\left(\frac{p}{q}\right)>1,
$$

since $m \geq 2$ and $\frac{p}{q}=\frac{1}{2^{x}}$

And $\sum_{u \in N(v)} f(u)=\underbrace{\frac{p}{q}+\frac{p}{q}+\ldots \ldots .+\frac{p}{q}}_{(m+2)-\text { times }}=(m+2)\left(\frac{p}{q}\right)>1$, since $m \geq 2$ and $\frac{p}{q}=\frac{1}{2^{x}}$

Again as in case 3 and 4 , we have that

$$
\sum_{u \in N(v)} f(u)=\underbrace{\frac{p}{q}+\frac{p}{q}+\ldots \ldots .+\frac{p}{q}}_{(m+1) \text {-times }}=(m+1)\left(\frac{p}{q}\right)>1,
$$

since $m \geq 2$ and $\frac{p}{q}=\frac{1}{2^{x}}$

$$
\text { And } \sum_{u \in N(v)} f(u)=\frac{p}{q}+\frac{p}{q}=2 \frac{\mathrm{p}}{\mathrm{q}}=2 \frac{1}{2}=1
$$

Therefore for all possibilities,

we get $\sum_{u \in N(v)} f(u) \geq 1$, $\mathbb{\forall} \in \mathbb{W}$.
This implies that $f$ is a TDF.

Now we check for the minimality of $f$.

Define $g: V \rightarrow[0,1]$ by

$g(v)= \begin{cases}r, & \text { if } v=v_{\mathbb{E}} \in V, \\ \frac{p}{q}, & \text { otherwise. }\end{cases}$

where $0<x<\frac{p}{q}$.

Since strict inequality holds at a vertex $\mathbb{E}_{\mathbb{R}}$ of $\mathrm{V}$, it follows that $\theta<f$.

Then we can show as in case (i) and (ii) of case 5 that

$$
\sum_{u \in N(v)} g(u)>1 \text {, if } \mathbb{v} \in \mathbb{P}_{n} \text { and } v_{k} \in N(v) \text { or } v_{k} \notin N(v) \text {. }
$$

Again as in case (iii) of case 5, we can show that

$\sum_{u \in N(v)} g(u)>1$, if $\mathbb{v} \in K_{1, m}$ and $v_{k} \in N(v)$ or $v_{k} \notin N(v)$.

As in case (iv) of case 5, we can show that

$$
\sum_{u \in N(v)} g(u)=r+\frac{p}{q}<2 \frac{\mathrm{p}}{\mathrm{q}}=2 \frac{1}{2}=1,
$$

if $\mathbb{v} \in K_{1 m}$ and $v_{k} \in N(v)$.

And $\sum_{u \in N(v)} g(u)=\frac{p}{q}+\frac{p}{q}=2 \frac{\mathrm{p}}{\mathrm{q}}=2 \frac{1}{2}=1$,

if $\mathbb{D} \in K_{1 \mathrm{~m}}$ and $v_{k} \notin N(v)$.

This implies that $\sum_{u \in N(v)} g(u)<1$, for some $\mathrm{v} \in \mathrm{V}$.

So $g$ is not a TDF.

Since $g$ is defined arbitrarily, it follows that there exists no $g<f$ such that $g$ is a TDF.

Thus $f$ is a MTDF.

\section{CONCLUSION}

It is interesting to study the total dominating functions of the corona product graph of a path with a star. This work gives the scope for the study of convexity of these minimal total dominating functions and the authors have also studied this concept. 


\section{ILLUSTRATION}

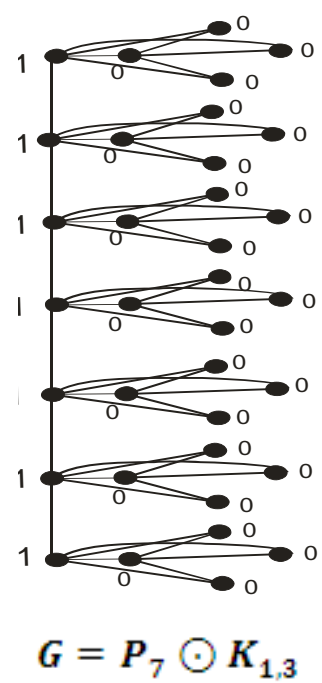

The function $\mathbf{f}$ takes the value 1 for the vertices of $P_{7}$ and the value 0 for the vertices in each copy of $K_{1,3}$.

\section{REFERENCES}

[1] Allan, R.B. and Laskar, R.C. - On domination, independent domination numbers of a graph. Discrete Math., 23 (1978), 73 - 76.

[2] Cockayne, E.J. and Hedetniemi, S.T. - Towards a theory of domination in graphs. Networks, 7 (1977), 247 - 261.

[3] Cockayne, C.J., Dawes, R.M. and Hedetniemi, S.T- Total domination in graphs, Networks, 10 (1980), 211 - 219.

[4] Cockayne, E.J., Mynhardt, C.M. and Yu, B.Total dominating functions in trees: Minimality and Convexity, Journal of Graph Theory, 19(1995), 83-92.
[5] Frucht, R. and Harary, F. - On the corona of Two Graphs, Aequationes Mathematicae, Volume 4, Issue 3 (1970), $322-325$.

[6] Haynes, T.W., Hedetniemi, S.T. and Slater, P.J. Domination in Graphs: Advanced Topics, Marcel Dekker, Inc., New York, (1998).

[7] Haynes, T.W., Hedetniemi, S.T. and Slater, P.J. Fundamentals of domination in graphs, Marcel Dekker, Inc., New York , (1998).

[8] Jeelani Begum, S. - Some studies on dominating functions of Quadratic Residue Cayley Graphs, $\mathrm{Ph}$. D. thesis, Sri Padmavathi Mahila Visvavidyalayam, Tirupati, Andhra Pradesh, India, (2011).

[9] Siva Parvathi, M - Some studies on dominating functions of corona product graphs, Ph.D thesis, Sri Padmavati Mahila Visvavidyalayam, Tirupati, Andhra Pradesh,India, (2013).

[10] Siva Parvathi, M and Maheswari, B .- Minimal Dominating Functions of Corona Product Graph of a Cycle with a Complete Graph - International Journal of Computer Engineering \& Technology, Volume 4, Issue 4 (2013), $248-256$.

[11] Siva Parvathi, M and Maheswari, B. - Some variations of Y-Dominating Functions of Corona Product Graph of a Cycle with a Complete Graph - International Journal of Computer Applications, Volume 81, Issue 1 (2013), 16 -21 .

[12] Siva Parvathi, M and Maheswari, B. - Some variations of Total Y-Dominating Functions of Corona Product Graph of a Cycle with a Complete Graph - Fire Journal of Science and Technology (accepted). 